\title{
ARTICLE
}

Received 17 Apr 2014 | Accepted 28 Aug 2014 | Published 5 Nov $2014 \quad$ DOl: 10.1038/ncomms6100

\section{Fano resonance and dipolar relaxation in lead-free relaxors}

D. Wang ${ }^{1}$, J. Hlinka ${ }^{2}$, A.A. Bokov ${ }^{3}$, Z.-G. Ye ${ }^{1,3}$, P. Ondrejkovic ${ }^{2}$, J. Petzelt ${ }^{2} \&$ L. Bellaiche ${ }^{4}$

Fano resonance is a phenomenon in which a discrete state interferes with a continuum of states and has been observed in many areas of science. Here, we report on the prediction of a Fano resonance in ferroelectric relaxors, whose properties are poorly understood: an ab initio molecular dynamic scheme reveals such resonance between the bare optical phonon mode of the $\mathrm{Zr}$ sublattice (the discrete state) and the bare optical phonon mode of the Ti sublattice (the continuum of states) in disordered lead-free $\mathrm{Ba}(\mathrm{Zr}, \mathrm{Ti}) \mathrm{O}_{3}$. The microscopic origins of the discrete state and continuum of states are discussed in the context of relaxor properties. Furthermore, our simulations suggest that the $T^{\star}$ characteristic temperature of relaxor is related to a hardening of the vibrational frequencies associated with fluctuation of the $\mathrm{Ti}$ sublattice. Finally, a terahertz relaxation mode reflecting reorientations of $\mathrm{Ti}$ dipoles and showing a thermally activated behaviour is predicted, in agreement with previous experiments.

\footnotetext{
${ }^{1}$ Electronic Materials Research Laboratory, Key Laboratory of the Ministry of Education and International Center for Dielectric Research, Xi'an Jiaotong University, Xi'an 710049, China. ${ }^{2}$ Institute of Physics, Academy of Sciences of the Czech Republic, Na Slovance 2, CZ-182 21 Praha 8, Czech Republic. ${ }^{3}$ Department of Chemistry and 4D LABS, Simon Fraser University, Burnaby, British Columbia, Canada V5A 1A6. ${ }^{4}$ Department of Physics, Institute for Nanoscience and Engineering, University of Arkansas, Fayetteville, Arkansas 72701, USA. Correspondence and requests for materials should be addressed to D.W. (email: dawei.wang@mail.xjtu.edu.cn).
} 
C anonical relaxor ferroelectrics are macroscopically centrosymmetric solid solutions or disordered compounds that are characterized by an unusually broad and frequency-dependent dielectric response versus temperature function ${ }^{1-32}$, which is often attributed to the hypothetical existence of polar nanoregions (PNRs) inside a paraelectric matrix. Relaxor ferroelectrics also typically exhibit three characteristic temperatures, known as the Burns temperature $T_{\mathrm{B}}$, freezing temperature $T_{\mathrm{f}}$ and the so-called $T^{\star}$ (refs $2,3,4,23,24$ ). While it is traditionally believed that $T_{\mathrm{B}}$ is associated with the formation of dynamical PNRs and that $T_{\mathrm{f}}$ corresponds to the temperature at which these PNRs become static, the precise microscopic origin of the third and intermediate critical temperature (that is $T^{\star}$ ) remains unclear and is intensively debated $^{24,33-35}$. Owing to their fundamental interest and also technological promise, relaxors have been studied by various techniques since their discoveries. For instance, they have been investigated by Raman, neutron elastic diffuse scattering, extended X-ray absorption fine structure, high-resolution tunnelling electron microscopy, piezoresponse force microscopy, phenomenology and numerical simulations $7,13,19,20,26,27,36-41$. On the other hand, there is an important aspect of their properties that has been much less documented because of experimental and computational challenges to overcome, that is, their terahertz $(\mathrm{THz})$ dynamics $25,27,42,43$. As a result, several issues remain poorly understood or even unknown. For instance, can different types of alloying atoms present in a relaxor (for example, $\mathrm{Zr}$ and $\mathrm{Ti}$ in $\mathrm{Ba}(\mathrm{Zr}, \mathrm{Ti}) \mathrm{O}_{3}(\mathrm{BZT})$ ) be associated with phonons having different dynamics? If it is indeed the case, can these different dynamics couple to each other and what is the underlying mechanism responsible for such hypothetical coupling? One may also wonder whether both alloyed sublattices, or only the one associated with the formation of PNRs (for example, Ti in BZT), exhibit relaxational modes inherent to the reorientation of dipoles? Another important challenge is to establish whether there is any inherent connection between characteristics of these phonon or relaxational modes and $T^{*}$, in order to determine the driving mechanism responsible for this temperature.

Obviously, answering all these questions would lead to a significant advance in our current understanding of relaxor ferroelectrics. In this study, all the aforementioned questions are addressed by investigating, analysing and comparing with experiments the complex dielectric response of disordered BZT crystal numerically obtained in the $\mathrm{THz}$ regime, via a combination of a first-principles-based effective Hamiltonian and a molecular dynamics (MD) technique. BZT is chosen because it is a lead-free relaxor that has already attracted a lot of attention $19,27,38,44-48$. In relaxors, most experimental works were performed in the kilohertz regime, which is (unfortunately) not accessible by our MD techniques. However, the dielectric relaxation in relaxors is typically observed in a much wider frequency interval, which is practically limited by the phonon frequencies from the high-frequency side. Because of extremely large dielectric permittivity of relaxors, the measurements in the gigahertz $(\mathrm{GHz})-\mathrm{THz}$ range are challenging, and very few experimental data are currently available. Therefore, the information that can be obtained from MD simulations is especially important. One particular feature of BZT is that it is also experimentally known ${ }^{28}$ to demonstrate a separate relaxation mechanism at the frequencies that are in the order of a few $\mathrm{cm}^{-1}$, which are accessible by the present simulations. BZT has also been selected because recent simulations did reveal the importance of PNRs (that are Ti rich), and the existence of all three known characteristic temperatures of relaxors (namely, $T_{\mathrm{B}}$ $\simeq 450 \mathrm{~K}, T^{*} \simeq 240 \mathrm{~K}$ and $\left.T_{\mathrm{f}} \simeq 130 \mathrm{~K}\right)$ in this system ${ }^{26,30,31}$.
In this article, we reveal the occurrence of a Fano resonance ${ }^{49}$ between phonon modes associated with the different types of alloyed atoms. We note that Fano resonance is a general phenomenon that is rather well known in, for example, nonlinear optics and photonics, but less documented in materials science. The Fano resonance is characterized by a specific interaction between a discrete state and a continuum of states $^{49-53}$. Moreover, $T^{\star}$ is found to be the temperature below which the vibrational frequencies associated with the fluctuation of the Ti sublattice significantly harden. This manuscript also reports the existence of a thermally activated relaxational mode (as consistent with experiments ${ }^{28}$ ) arising from the reorientation of $\mathrm{Ti}$ dipoles.

\section{Results}

Dielectric response. The studied material with the composition of $\mathrm{Ba}\left(\mathrm{Zr}_{0.5} \mathrm{Ti}_{0.5}\right) \mathrm{O}_{3}$ is made of $50 \%$ of $\mathrm{Zr}$ and $\mathrm{Ti}$ ions that are randomly distributed inside a $12 \times 12 \times 12$ supercell $(8,640$ atoms). The latter is periodic along the $x, y$ and $z$ axes, which lie along the pseudocubic [100], [010] and [001] directions, respectively. We perform effective Hamiltonian-based MD simulations $^{54-57}$ to obtain complex dielectric responses of disordered $\mathrm{BZT}$ for temperatures ranging from 10 to $1,000 \mathrm{~K}$ (see the Methods section). At the end of the MD simulations, the complex electric susceptibility, $\chi_{\alpha \beta}(v)$, can be obtained using the following equation $^{56-59}$ :

$$
\chi_{\alpha \beta}(v)=\frac{1}{\varepsilon_{0} V k_{\mathrm{B}} T}\left[\left\langle\mathbf{d}_{\alpha}(t) \mathbf{d}_{\beta}(t)\right\rangle+i 2 \pi v \int_{0}^{\infty} \mathrm{d} t e^{i 2 \pi v t}\left\langle\mathbf{d}_{\alpha}(t) \mathbf{d}_{\beta}(0)\right\rangle\right]
$$

where $v$ is the frequency while $\alpha$ and $\beta$ define Cartesian components, $V$ is the volume of the chosen supercell and $\varepsilon_{0}$ is the vacuum permittivity. $\mathbf{d}(t)$ is the electric dipole moment of the system at time $t$, and $\langle\ldots\rangle$ represents thermal averages.

The total electric dipole moment, $\mathbf{d}$, of the system in equation (1) obviously consists of two parts $\mathbf{d}=\mathbf{d}^{Z \mathbf{r}}+\mathbf{d}^{\mathrm{Ti}}$ where $\mathbf{d}^{\mathrm{Zr}}$ (respectively, $\mathbf{d}^{\mathrm{Ti}}$ ) is the dipole moment originating from the electric dipoles centred on $\mathrm{Zr}$ (respectively, Ti) sites. As a result, the total dielectric response of equation (1) can be decomposed additively into three different contributions:

$$
\begin{aligned}
\chi_{\alpha \beta}^{\mathrm{Zr}, \mathrm{Zr}}(v)= & \frac{1}{\varepsilon_{0} V k_{\mathrm{B}} T}\left[\left\langle\mathbf{d}_{\alpha}^{\mathrm{Zr}}(t) \mathbf{d}_{\beta}^{\mathrm{Zr}}(t)\right\rangle+i 2 \pi v \int_{0}^{\infty} \mathrm{d} t e^{i 2 \pi v t}\left\langle\mathbf{d}_{\alpha}^{\mathrm{Zr}}(t) \mathbf{d}_{\beta}^{\mathrm{Zr}}(0)\right\rangle\right], \\
\chi_{\alpha \beta}^{\mathrm{Ti}, \mathrm{Ti}}(v)= & \frac{1}{\varepsilon_{0} V k_{\mathrm{B}} T}\left[\left\langle\mathbf{d}_{\alpha}^{\mathrm{Ti}}(t) \mathbf{d}_{\beta}^{\mathrm{Ti}}(t)\right\rangle+i 2 \pi v \int_{0}^{\infty} \mathrm{d} t e^{i 2 \pi v t}\left\langle\mathbf{d}_{\alpha}^{\mathrm{Ti}}(t) \mathbf{d}_{\beta}^{\mathrm{Ti}}(0)\right\rangle\right], \\
\chi_{\alpha \beta}^{\mathrm{Ti}, \mathrm{Zr}}(v)= & \frac{1}{\varepsilon_{0} V k_{\mathrm{B}} T}\left\{\left\langle\mathbf{d}_{\alpha}^{\mathrm{Ti}}(t) \mathbf{d}_{\beta}^{\mathrm{Zr}}(t)\right\rangle+\left\langle\mathbf{d}_{\alpha}^{\mathrm{Zr}}(t) \mathbf{d}_{\beta}^{\mathrm{Ti}}(t)\right\rangle+i 2 \pi v\right. \\
& \left.\int_{0}^{\infty} \mathrm{d} t e^{i 2 \pi v t}\left[\left\langle\mathbf{d}_{\alpha}^{\mathrm{Ti}}(t) \mathbf{d}_{\beta}^{\mathrm{Zr}}(0)\right\rangle+\left\langle\mathbf{d}_{\alpha}^{\mathrm{Zr}}(t) \mathbf{d}_{\beta}^{\mathrm{Ti}}(0)\right\rangle\right]\right\} .
\end{aligned}
$$

Here, $\chi_{\alpha \beta}^{\mathrm{Zr}, \mathrm{Zr}}$ and $\chi_{\alpha \beta}^{\mathrm{Ti}, \mathrm{Ti}}$ represent the 'sole' contributions of the Zr-centred sites and Ti-centred sites, respectively, to the total dielectric response. On the other hand, $\chi_{\alpha \beta}^{\mathrm{Ti}, \mathrm{Zr}}$ quantifies the role of the correlations between the $\mathrm{Zr}$ and $\mathrm{Ti}$-centred sites in that total response. Owing to the complexity of the system under investigation, we found it is more convenient and instructive to 
separately compute (and fit) $\chi_{\alpha \beta}^{\mathrm{Zr}, \mathrm{Zr}}, \chi_{\alpha \beta}^{\mathrm{Ti}, \mathrm{Ti}}$ and $\chi_{\alpha \beta}^{\mathrm{Ti}, \mathrm{Zr}}$ rather than the total dielectric response. The latter can then be deduced by combining the fittings of $\chi_{\alpha \beta}^{\mathrm{Zr}, \mathrm{Zr}}, \chi_{\alpha \beta}^{\mathrm{Ti}, \mathrm{Ti}}$ and $\chi_{\alpha \beta}^{\mathrm{Ti}, \mathrm{Zr}}$, as shown in Fig. 1 for the imaginary part of these responses. Note that, in the following, we will focus on the isotropic component (that is, the average of the $x x, y y$ and $z z$ components) of each of these different dielectric responses, but will not use any notation having subscript for brevity.

Fano resonance. Let us first concentrate on the MD data for the imaginary part of these different dielectric responses at $T=900 \mathrm{~K}$ (see first row of Fig. 1). The imaginary part of $\chi^{\mathrm{Zr}, \mathrm{Zr}}$ consists of a single narrow peak centred at $\sim 155 \mathrm{~cm}^{-1}$. On the other hand, both the imaginary parts of $\chi^{\mathrm{Ti}, \mathrm{Ti}}$ and $\chi^{\mathrm{Ti}, \mathrm{Zr}}$ exhibit anomalous features. As a matter of fact, $\operatorname{Im}\left(\chi^{\mathrm{Ti}, \mathrm{Ti}}\right)$ appears to have two (rather than one) significant broad peaks centred at $\sim 130$ and $250 \mathrm{~cm}^{-1}$, respectively. Similarly striking, the imaginary part of $\chi^{\mathrm{Ti}, \mathrm{Zr}}$ possesses a narrow peak of $\sim 160 \mathrm{~cm}^{-1}$ (like $\chi^{Z r, Z r}$ ) that is followed by negative values for frequencies ranging from 200 to $300 \mathrm{~cm}^{-1}$, which also results in the asymmetry of the lineshape (Fig. 1c).

Interestingly, since refs. 26,44 indicated that PNRs (inside which $\mathrm{Ti}$ dipoles are parallel to each other) begin to form in BZT at the Burns temperature $T_{\mathrm{B}} \simeq 450 \mathrm{~K}$, there are no PNRs at $900 \mathrm{~K}$. As a result, the anisotropy introduced by PNRs cannot be the cause of the two peaks seen in $\operatorname{Im}\left(\chi^{\mathrm{Ti}, \mathrm{Ti}}\right)$ at $900 \mathrm{~K}$. A mechanism that does not involve PNRs must therefore be responsible for the unusual features of $\chi^{\mathrm{Ti}, \mathrm{Ti}}$ and $\chi^{\mathrm{Ti}, \mathrm{Zr}}$. Here, we take advantage of the insight brought by our simulations to reveal that a Fano resonance naturally explains such unusual features.

For that, let us consider two linearly coupled damped harmonic oscillators (DHOs) - one of them (with bare frequency $v_{\mathrm{Ti}, 0}$ and bare damping constant $\Gamma_{\mathrm{Ti}, 0}$ ) representing the intrinsic, bare dynamical response of the Ti sublattice, and the other (with bare frequency $v_{\mathrm{Zr}, 0}$ and bare damping constant $\left.\Gamma_{\mathrm{Zr}, 0}\right)$ representing the intrinsic, bare dynamical response of the $\mathrm{Zr}$ sublattice. The dynamics of the coupled system can be described by a $2 \times 2$ Green's function $\mathbf{G}(v)=\left(\mathbf{D}-i v \boldsymbol{\Gamma}-v^{2} \mathbf{I}\right)^{-1}$ (refs 57,60$)$ where $\mathbf{I}$ is the $2 \times 2$ unit matrix, while the real dynamical matrix $\mathbf{D}$ and the real damping parameter matrix $\Gamma$ read

$$
\mathbf{D}=\left(\begin{array}{cc}
v_{\mathrm{Ti}, 0}^{2} & \Delta_{\mathrm{Ti}, \mathrm{Zr}} \\
\Delta_{\mathrm{Ti}, \mathrm{Zr}} & v_{\mathrm{Zr}, 0}^{2}
\end{array}\right), \boldsymbol{\Gamma}=\left(\begin{array}{cc}
\Gamma_{\mathrm{Ti}, 0} & \Gamma_{\mathrm{Ti}, \mathrm{Zr}} \\
\Gamma_{\mathrm{Ti}, \mathrm{Zr}} & \Gamma_{\mathrm{Zr}, 0}
\end{array}\right) .
$$

These matrices contain off-diagonal terms $\Delta_{\mathrm{Ti}, \mathrm{Zr}}$ and $\Gamma_{\mathrm{Ti}, \mathrm{Zr}}$ describing the bilinear coupling between the $\mathrm{Ti}$ and $\mathrm{Zr}$ sublattices. Contributions to the complex dielectric susceptibility spectrum $\chi(v)$ of this coupled system can be expressed via (bare) plasma frequencies $\Omega_{\mathrm{Ti}, 0}$ and $\Omega_{\mathrm{Zr}, 0}$ (for example, ref. 61), associated with the total dipole moment of the $\mathrm{Ti}$ and $\mathrm{Zr}$ subsystems, respectively, as $\chi^{\mathrm{Ti}, \mathrm{Ti}}=\left(\Omega_{\mathrm{Ti}, 0}, 0\right) \mathbf{G}(v)\left(\begin{array}{c}\Omega_{\mathrm{Ti}, 0} \\ 0\end{array}\right), \chi^{\mathrm{Zr}, \mathrm{Zr}}=$ $\left(0, \Omega_{\mathrm{Zr}, 0}\right) \mathbf{G}(v)\left(\begin{array}{c}0 \\ \Omega_{\mathrm{Zr}, 0}\end{array}\right), \quad$ and $\quad \chi^{\mathrm{Ti}, \mathrm{Zr}}=\left(\Omega_{\mathrm{Ti}, 0}, 0\right) \mathbf{G}(v)\left(\begin{array}{c}0 \\ \Omega_{\mathrm{Zr}, 0}\end{array}\right)+$
C
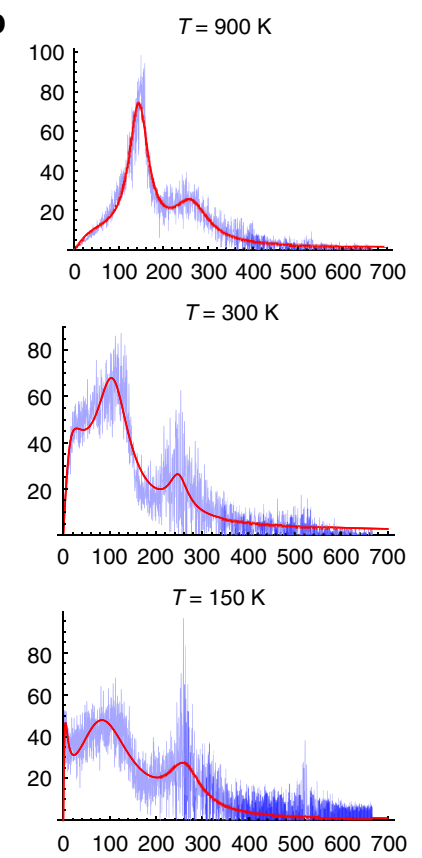

$T=20 \mathrm{~K}$

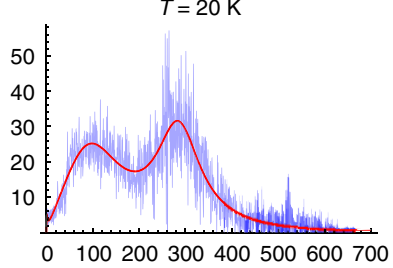

C

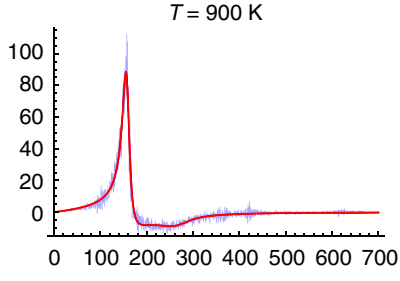

$T=300 \mathrm{~K}$

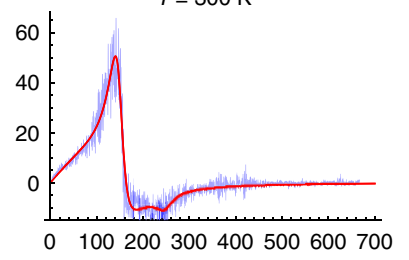

$T=150 \mathrm{~K}$

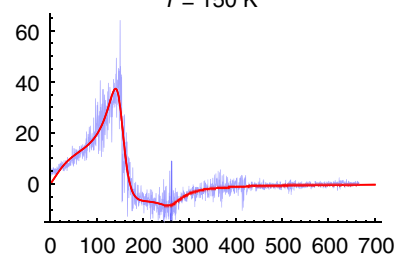

$T=20 \mathrm{~K}$

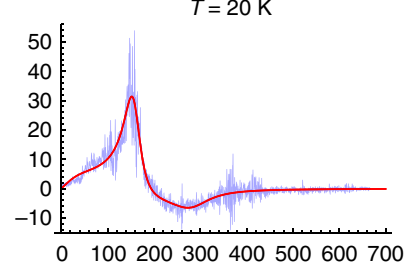

d
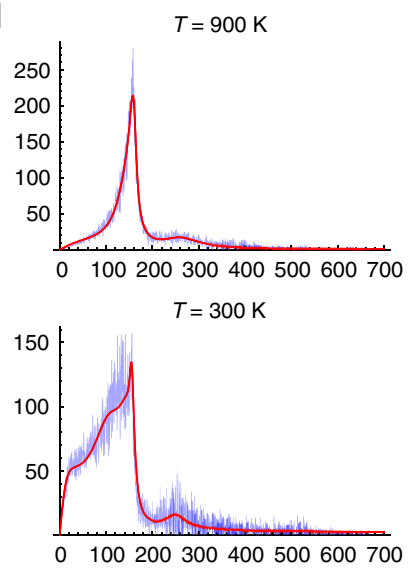

$T=150 \mathrm{~K}$
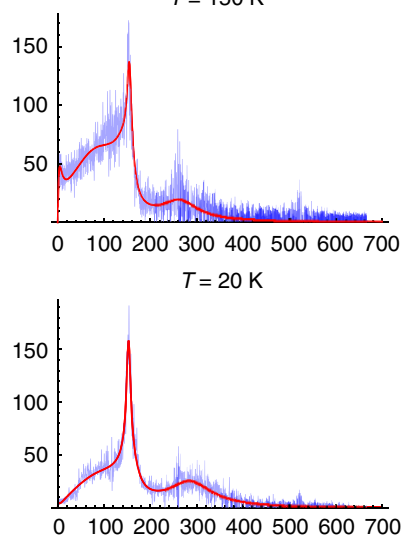

Frequency $\left(\mathrm{cm}^{-1}\right)$

Figure 1 | Imaginary part of the dielectric responses of BZT as a function of frequency. (a) The dielectric response from $Z r$ cells, $\chi^{Z r, Z r}$. (b) The dielectric response from Ti cells, $\chi^{\mathrm{Ti}, \mathrm{T}}$. (c) The dielectric response from the cross-correlation between $\mathrm{Ti}$ and $\mathrm{Zr}$ cells, $\chi^{\mathrm{Ti}, Z \mathrm{r}}$. (d) The total dielectric response. The solid lines in these panels represent the fitting of the MD data using analytical expressions given in equations (6) and (7), and $Q(v)$. The actual MD data are also shown (in blue colour) in order to confirm the adequacy of the fittings. 
$\left(0, \Omega_{\mathrm{Zr}, 0}\right) \mathbf{G}(v)\left(\begin{array}{c}\Omega_{\mathrm{Ti}, 0} \\ 0\end{array}\right)$. Within this coupled DHOs model, the bare dielectric susceptibilities of the $\mathrm{Zr}$ and Ti oscillators $\left(\chi_{0}^{\mathrm{Zr}, \mathrm{Zr}}, \chi_{0}^{\mathrm{Ti}, \mathrm{Ti}}\right)$ are given by $\chi_{0}^{\mathrm{Zr}, \mathrm{Zr}}=\Omega_{\mathrm{Zr}, 0}^{2} /\left(v_{\mathrm{Zr}, 0}^{2}-i v \Gamma_{\mathrm{Zr}, 0}-v^{2}\right)$ and $\chi_{0}^{\mathrm{Ti}, \mathrm{Ti}}=\Omega_{\mathrm{Ti}, 0}^{2} /\left(v_{\mathrm{Ti}, 0}^{2}-i v \Gamma_{\mathrm{Ti}, 0}-v^{2}\right)$, which can also be expressed in terms of the (perturbed) partial susceptibilities as $\quad \chi_{0}^{\mathrm{Zr}, \mathrm{Zr}}=\chi^{\mathrm{Zr}, \mathrm{Zr}}-\left(\chi^{\mathrm{Ti}, \mathrm{Zr}}\right)^{2} / 4 \chi^{\mathrm{Ti}, \mathrm{Ti}} \quad$ and $\quad \chi_{0}^{\mathrm{Ti}, \mathrm{Ti}}=\chi^{\mathrm{Ti}, \mathrm{Ti}}-$ $\left(\chi^{\mathrm{Ti}, Z \mathrm{Zr}}\right)^{2} / 4 \chi^{\mathrm{Zr}, Z \mathrm{Zr}}$.

Application of this model to the simulated dielectric response at $900 \mathrm{~K}$ is shown in Fig. 2 as an example. In this case, the parameters entering $\chi^{\mathrm{Zr}, \mathrm{Zr}}$ and $\chi^{\mathrm{Ti}, \mathrm{Ti}}$, which best fit the $\mathrm{MD}$ results of $\chi^{\mathrm{Zr}, \mathrm{Zr}}$ and $\chi^{\mathrm{Ti}, \mathrm{Ti}}$ simultaneously (shown in Fig. 2a,b), respectively, are such as: (i) the bare $\mathrm{Zr}$ oscillator is described by $\Omega_{\mathrm{Zr}, 0}=450 \mathrm{~cm}^{-1}, v_{\mathrm{Zr}, 0}=170 \mathrm{~cm}^{-1}$ and $\Gamma_{\mathrm{Zr}, 0}=19$ $\mathrm{cm}^{-1}$; (ii) the bare Ti oscillator is described by $\Omega_{\mathrm{Ti}, 0}=1,250$ $\mathrm{cm}^{-1}, v_{\mathrm{Ti}, 0}=193 \mathrm{~cm}^{-1}$ and $\Gamma_{\mathrm{Ti}, 0}=205 \mathrm{~cm}^{-1}$; and (iii) the coupling constants are $\Delta_{\mathrm{Ti}, \mathrm{Zr}}=-8,435 \mathrm{~cm}^{-1}$ and $\Gamma_{\mathrm{Ti}, \mathrm{Zr}}=-41$ $\mathrm{cm}^{-1}$. The resulting bare $\chi_{0}^{\mathrm{Zr}, \mathrm{Zr}}$ and $\chi_{0}^{\mathrm{Ti}, \mathrm{Ti}}$, associated with these parameters, are shown in Fig. 2 d. Note that these $\Omega_{\mathrm{Ti}, 0}$ and $\Omega_{\mathrm{Zr}, 0}$ plasma frequencies correspond well to anticipated dynamical charge associated with the $\mathrm{Ti}$ and $\mathrm{Zr}$ sublattices of the effective Hamiltonian model. Furthermore, it is important to realize that the bare $\mathrm{Ti}$ oscillator is clearly much more damped than the $\mathrm{Zr}$ one. One therefore recovers a broad spectrum for the bare $\mathrm{Ti}$ subsystem overlapping with the resonant bare response of the $\mathrm{Zr}$ subsystem. From these comparisons, it is clear that the two asymmetric peaks in $\chi^{\mathrm{Ti}, \mathrm{Ti}}$ are indeed caused by an interaction

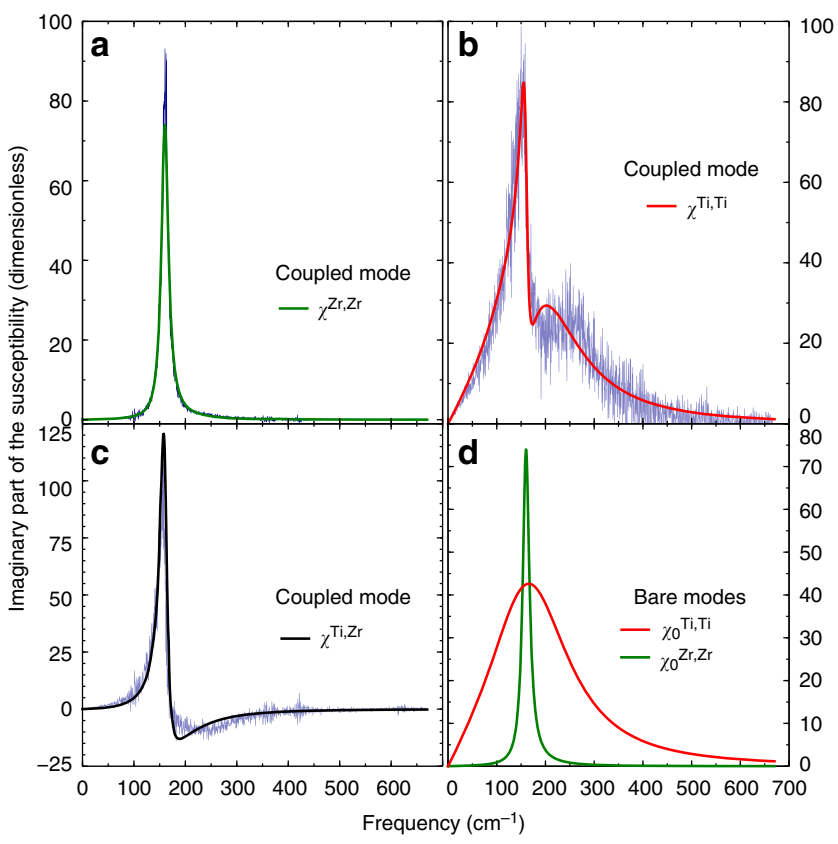

Figure 2 | Imaginary part of different dielectric responses of BZT as a function of frequency at $\boldsymbol{T}=\mathbf{9 0 0} \mathbf{K}$. (a-c) The dielectric responses from $Z r$ cells, $\chi^{Z r, Z r}$, from Ti cells, $\chi^{\mathrm{Ti}, \mathrm{Ti}}$, and from the cross-correlation

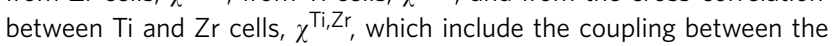
bare responses of $\mathrm{Ti}$ and $\mathrm{Zr}$ sublattices. (d) The bare dielectric response from $Z r$ cells, $\chi_{0}^{Z r, Z r}$ and bare dielectric response from Ti cells, $\chi_{0}^{\mathrm{Ti}, T i}$. The actual MD data are also shown (in blue colour) in order to confirm that Fano interference leads to the main striking features adopted by $\chi^{\mathrm{Ti}, \mathrm{Ti}}$ and $\chi^{\mathrm{Ti}, Z \mathrm{r}}$. The parameters entering this interference are found by simultaneously fitting the MD data of $\chi^{\mathrm{Ti}, \mathrm{Ti}}$ and $\chi^{\mathrm{Zr}, \mathrm{Zr}}$ using

$\chi^{\mathrm{Ti}, \mathrm{Ti}}=\left(\Omega_{\mathrm{Ti}, 0}, 0\right) \mathbf{G}(v)\left(\begin{array}{c}\Omega_{\mathrm{T}, 0} \\ 0\end{array}\right)$ and $\chi^{\mathrm{Zr}, \mathrm{Zr}}=\left(0, \Omega_{\mathrm{Zr}, 0}\right) \mathbf{G}(v)\left(\begin{array}{c}0 \\ \Omega_{\mathrm{Zr}, 0}\end{array}\right)$. between the $\mathrm{Ti}$ and $\mathrm{Zr}$ subsystems. Note that the difference between $\Gamma_{\mathrm{Ti}, 0}$ and $\Gamma_{\mathrm{Zr}, 0}$ is compatible with the Fano resonance mechanism, that is, the broad bare $\mathrm{Ti}$ response provides the background component while the sharp bare $\mathrm{Zr}$ response provides the discrete state, the two ingredients for the Fano resonance. To understand such difference at a microscopic level, let us recall that ref. 26 revealed that $\mathrm{Zr}$ ions are close to their ideal cubic positions, leading to a very narrow peak in their bare dielectric response. On the other hand, the Ti ions move away from the ideal cubic positions and have an underlying anharmonic potential, with the amplitude of these motions depending on the chemical environment. As a result, the bare dielectric response of the Ti sublattice has a broad peak. Note also that the difference in the amplitude of the motions between the $\mathrm{Zr}$ and $\mathrm{Ti}$ ions was precisely found to be the origin of the formation of PNRs in disordered BZT ${ }^{26}$. In other words, the Fano resonance and the characteristic quantities of relaxors are found to have the same microscopic origin in our investigated disordered solid solution. Note, however, that some other properties of relaxors, such as the dependence of their susceptibility versus temperature function on frequency, are likely caused by the existence of relaxational modes (associated, for example, with dipolar dynamics of PNRs), and are thus not directly related to the Fano resonance since the latter involves phonon modes.

Moreover, and as further evidenced in Fig. $2 c$ (that displays the cross-function $\chi^{\mathrm{Ti}, \mathrm{Zr}}$, resulting from the aforementioned coupled DHO model and parameters, along with the actual MD data), the motions of the $\mathrm{Ti}$ and $\mathrm{Zr}$ ions become coupled to each other as a result of this Fano coupling. One can clearly see that the asymmetry and negative values of $\chi^{\mathrm{Ti}, \mathrm{Zr}}$, are all well reproduced by the fitting, which further emphasizes the relevance of the Fano resonance in BZT relaxors. Note that a Fano resonance has also been recently suggested to have a role in the $\left(\mathrm{K}_{1-{ }_{x}} \mathrm{Li}_{x}\right) \mathrm{TaO}_{3}$ relaxor ferroelectric ${ }^{53}$. However, this resonance is different from ours and rather complex, since it is proposed to involve normal modes (corresponding to different discrete oscillating configurations) of the whole nanocomposite (that is, made of the polar nanodomains and the host matrix) that are coherently coupled to each other via a piezoelectrically assisted relaxation continuum. Similarly, the Fano resonances observed in the nonrelaxor $\mathrm{LiNbO}_{3}$ material ${ }^{51}$ are certainly of different nature. In contrast, our proposed Fano resonance simply consists of two coupled optical phonon modes. To further demonstrate that the proposed Fano resonance occurs in BZT relaxors, we performed additional MD simulations in which the resonance of $\chi^{\mathrm{Zr}, \mathrm{Zr}}$ is artificially tuned to $450 \mathrm{~cm}^{-1}$, which is far away from the (bare) resonance of $\chi_{0}^{\mathrm{Ti}, \mathrm{Ti}}$. In such a case, we numerically found that, for example, $\chi^{\mathrm{Ti}, \mathrm{Ti}^{\mathrm{i}}}$ has now only one (broad) peak rather than two, which confirms once again the adequacy of the Fano interference in our investigated system.

The aforementioned simple model, based on a Fano interference between the bare DHO modes of the $\mathrm{Zr}$ and $\mathrm{Ti}$ sublattices, therefore allows us to understand and reproduce all the striking features of the perturbed dielectric spectra (that is, the single sharp peak of $\chi^{\mathrm{Zr}, \mathrm{Zr}}$, the double broad peaks of $\chi^{\mathrm{Ti}, \mathrm{Ti}}$, and the asymmetry and negative values of $\chi^{\mathrm{Ti}, \mathrm{Zr}}$. However, as shown in Fig. 2, this model does not 'perfectly' fit the MD data over the entire range of investigated frequencies. For instance, the second (highest-in-frequency) peak of $\chi^{\mathrm{Ti}, \mathrm{Ti}}$ could be better described between $\simeq 200$ and $300 \mathrm{~cm}^{-1}$. We numerically found that such slight imperfection is related to the atomic disorder existing inside our investigated system. This fact is evidenced by further simulations (Supplementary Note 1) we conducted at $900 \mathrm{~K}$ on a perfectly rocksalt-ordered realization of BZT in which the $\mathrm{Zr}$ and Ti ions alternate along any Cartesian direction (note that this rocksalt ordering is possible to create when the overall $\mathrm{Zr}$ and 
Ti compositions are both equal to 0.5 , as we choose here). In that case, coupling of the single DHOs for the bare responses of the $\mathrm{Zr}$ and Ti sublattices leads to an excellent fitting of the MD data (Supplementary Fig. 1).

Let us now investigate the $\chi^{\mathrm{Zr}, \mathrm{Zr}}, \chi^{\mathrm{Ti}, \mathrm{Ti}}$ and $\chi^{\mathrm{Ti}, \mathrm{Zr}}$ of the disordered BZT material for all temperatures, not only for high temperatures $(\sim 900 \mathrm{~K})$, for which the aforementioned Fano model is enough to reproduce and understand the MD data. We (empirically) found that these dielectric responses can, in fact, be reasonably well fitted for any temperature by different analytical expressions, which we now report and discuss. The sole contribution of the $\mathrm{Zr}$-centred sites, $\chi^{\mathrm{Zr}, \mathrm{Zr}}$, can be well fitted by a single $\mathrm{DHO}$ of the form:

$$
\chi^{\mathrm{Zr}, \mathrm{Zr}}=\frac{\Omega_{\mathrm{Zr}}^{2}}{v_{\mathrm{Zr}-\mathrm{Zr}}^{2}-v^{2}-i \gamma_{\mathrm{Zr}-\mathrm{Zr}} v},
$$

where $v_{\mathrm{Zr}-\mathrm{Zr}}$ is the resonant frequency, $\Omega_{\mathrm{Zr}}$ is the plasma frequency and $\gamma_{Z r-Z r}$ is the damping constant of this mode. Note that $\Omega_{\mathrm{Zr}}$ is numerically found to be approximately a constant independent of temperature, as consistent with the anticipated dynamical charge associated with the $\mathrm{Zr}$ sublattice of the effective Hamiltonian model. The result of this fitting of the imaginary part of $\chi^{\mathrm{Zr}, \mathrm{Zr}}$ is shown in Fig. 1a for four representative temperatures, which are $T=900,300,150$ and $20 \mathrm{~K}$, showing a narrow peak for any of these temperatures. Regarding $\chi^{\mathrm{Ti}, \mathrm{Ti}}$, we decided to use two DHOs and also a Debye mode for any temperature. The necessity of two DHOs for $\chi^{\mathrm{Ti}, T i}$ fitting is a consequence of the aforementioned Fano coupling between the bare DHO modes of $\mathrm{Zr}$ and $\mathrm{Ti}$ sublattices. The introduction of the Debye mode was deemed necessary in order to describe the low-frequency peak that can be clearly seen in Fig. $1 \mathrm{~b}$ for the 300 and $150 \mathrm{~K}$ data. It does not originate from, nor is involved in, the presently discovered Fano coupling, unlike the Fano mechanism proposed in $\left(\mathrm{K}_{1-x} \mathrm{Li}_{x}\right) \mathrm{TaO}_{3}$ (ref. 53). As we will show later, it is rather related to the flipping of Ti dipoles, which is typical of the so-called central mode ${ }^{56,57}$. We thus have:

$$
\chi^{\mathrm{Ti}, \mathrm{Ti}}=\frac{S_{\mathrm{Ti}-\mathrm{Ti}}^{r}}{1-i \tau_{\mathrm{Ti}-\mathrm{Ti}} v}+\sum_{i=1}^{2} \frac{\Omega_{\mathrm{Ti}, i}^{2}}{v_{\mathrm{Ti}-\mathrm{Ti}, i}^{2}-v^{2}-i \gamma_{\mathrm{Ti}-\mathrm{Ti}, i} v},
$$

where $\tau_{\mathrm{Ti}-\mathrm{Ti}}$ and $S_{\mathrm{Ti}-\mathrm{Ti}}^{r}$ are the relaxation time and static susceptibility of the Debye mode, respectively. $v_{\mathrm{Ti}-\mathrm{Ti}, i}, \Omega_{\mathrm{Ti}-\mathrm{Ti}, i}$ and $\gamma_{\mathrm{Ti}-\mathrm{Ti}, i}$ represent the resonant frequency, plasma frequency and damping constant of the Ti-DHO mode $i$ (with $i=1$ or 2 ), respectively. The notation $i=1$ (respectively, $i=2$ ) corresponds to the DHO mode having the smallest (respectively, largest) resonant frequency.

Figure 3 reports the temperature evolution of the resonant frequencies of all the DHO modes associated with the $\mathrm{Zr}$ and $\mathrm{Ti}$ dielectric responses, as well as the bare resonant frequency $v_{\mathrm{Ti}, 0}$ associated with $\chi_{0}^{\mathrm{Ti}, \mathrm{Ti}}$ (note that the bare resonant frequency $v_{\mathrm{Zr}, 0}$ associated with $\chi_{0}$, Zr is very similar to $v_{\mathrm{Zr}-\mathrm{Zr}}$, and is thus not shown for clarity). The resonant frequency $v_{\mathrm{Zr}-\mathrm{Zr}}$ very slightly varies between 160 and $152 \mathrm{~cm}^{-1}$ when the temperature decreases. $v_{\mathrm{Ti}-\mathrm{Ti}, 1}$ ranges from 110 to $150 \mathrm{~cm}^{-1}$ for the investigated temperature window. $v_{\mathrm{Ti}-\mathrm{Ti}, 2}$ only slightly decreases when decreasing the temperature down to $\sim 240 \mathrm{~K}$, and then significantly increases from 250 to $300 \mathrm{~cm}^{-1}$ as the temperature is further decreased. Interestingly, the existence of the three predicted DHO modes for the perturbed responses, as well as the value and temperature behaviours of their resonant frequencies shown in Fig. 3, is rather consistent with the experimental work on $\mathrm{Ba}\left(\mathrm{Zr}_{0.4} \mathrm{Ti}_{0.6}\right) \mathrm{O}_{3}$ (ref. 27), revealing the existence of two modes at $\sim 100$ and $140-150 \mathrm{~cm}^{-1}$ that are nearly independent of temperature and a third phonon mode at $200 \mathrm{~cm}^{-1}$ whose resonant frequency increases when the temperature decreases

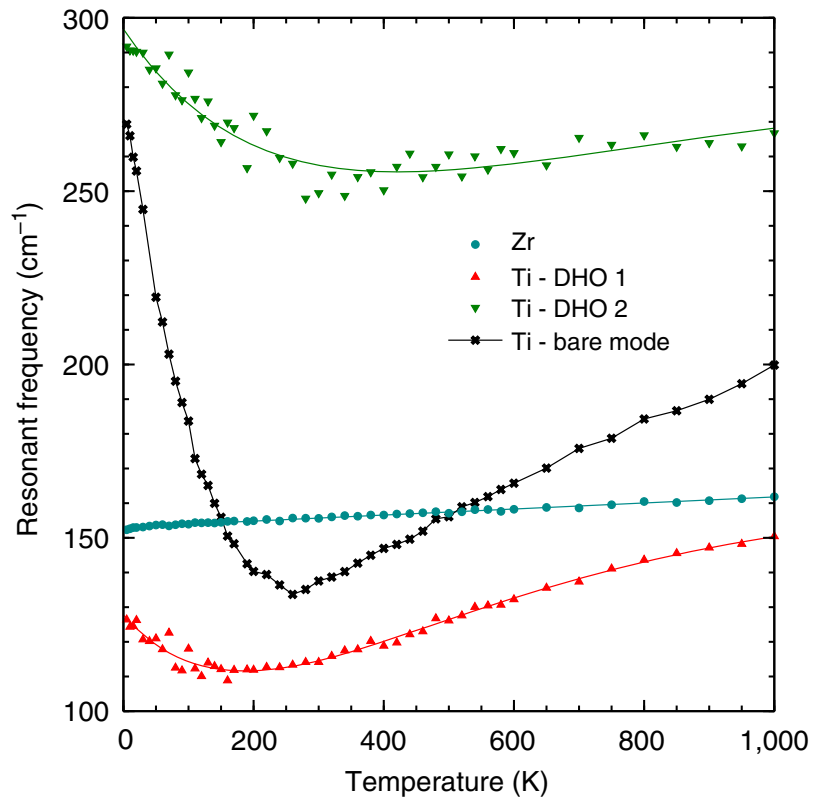

Figure 3 | Resonant frequencies of the DHO modes associated with the $\mathrm{Zr}$ and of Ti sublattices as a function of temperature. These modes correspond to the $\mathrm{Zr}$-DHO mode, the Ti-DHO mode 1, the Ti-DHO mode 2 and the bare Ti-mode.

below $250 \mathrm{~K}$. According to our simulations, such modes correspond to the Ti-DHO mode 1, the DHO mode of $\mathrm{Zr}$ ions and the Ti-DHO mode 2, respectively (note, however, that there is a subtle difference between the measurements and simulations, in the sense that the experiments indicate that the lowest transverse optical phonon mode of $\mathrm{Zr}$ ions and $\mathrm{Ti}$ ions should be of the Last and Slater type, respectively ${ }^{27}$, while the simulations lead to an intermediate situation between the Last and Slater types for the three aforementioned DHOs by construction of the effective Hamiltonian, see the Methods section). Figure 3 further reveals that the noticeable non-monotonic behaviour of $v_{\mathrm{Ti}-\mathrm{Ti}, 2}$ at $\sim 240 \mathrm{~K}$ originates from a striking enhancement of the bare resonant frequency $v_{\mathrm{Ti}, 0}$ below such temperature. Remarkably, $240 \mathrm{~K}$ is the previously predicted value of the so-called $T^{*}$ characteristic temperature ${ }^{44}$ of $\mathrm{BZT}^{26}$. Moreover, the average magnitude of the Ti dipoles was found to significantly increase when decreasing the temperature below $T^{*}$ in ref. 26, which bears resemblance to the enhancements of $v_{\mathrm{Ti}-\mathrm{Ti}, 2}$ from 250 to $300 \mathrm{~cm}^{-1}$ and of $v_{\mathrm{T}, 0}$ from 130 to $270 \mathrm{~cm}^{-1}$ depicted in Fig. 3. This controversial characteristic temperature $T^{*}$ therefore appears to be associated with a hardening of a very specific sublattice, namely the Ti sublattice. It will be interesting in the future to determine whether this is the case for other relaxors, since recent works seem to indicate that $T^{\star}$ may have a different origin between lead-free and lead-based relaxors ${ }^{33-35}$.

Dipolar relaxation. As indicated in equation $(7), \chi^{\mathrm{Ti}, \mathrm{Ti}}$ also possesses a relaxation mode in addition to these two DHO modes. Microscopically, the existence of DHOs and Debye mode is because of the pronounced multipotential well structure for the dipoles on Ti sites: DHO modes are typically related to oscillations of Ti ions inside potential wells, whereas the Debye mode is associated with the hopping of Ti ions among the off-centred sites, which is also known as the central mode ${ }^{56,57}$. This relaxation mode is denoted as $R_{\mathrm{Ti}-\mathrm{Ti}}$ in the following. As shown in Fig. $4 \mathrm{a}$, the Debye frequency of $R_{\mathrm{Ti}-\mathrm{Ti}}$ (that is, $1 / \tau_{\mathrm{Ti}-\mathrm{Ti}}$ ) 
a

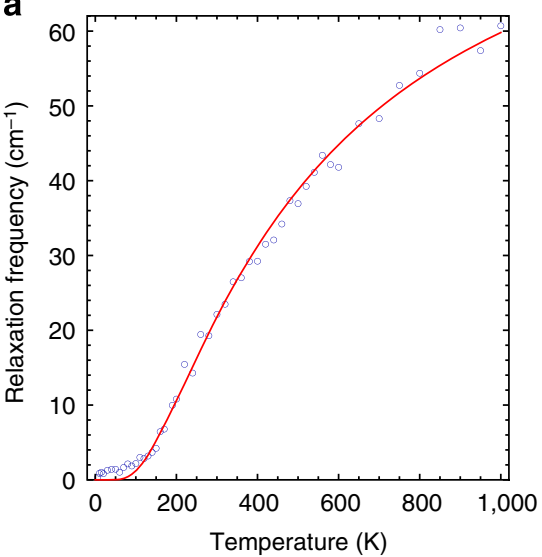

b

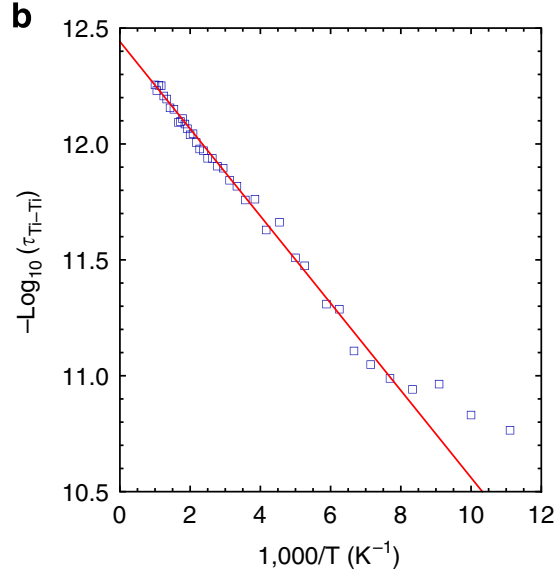

Figure 4 | Temperature dependence of the Debye frequency associated with the $\boldsymbol{R}_{\mathbf{T} \text {-Ti }}$ relaxational mode of Ti ions. (a) The dependence in linear scales is shown in $\mathbf{a}$, while in $\mathbf{b}$ such frequency is shown in logarithmic scale with $\tau_{\mathrm{Ti}-\mathrm{Ti}}$ being in unit of second, and as a function of the ratio of 1,000 and the temperature (expressed in Kelvin). The solid line in both panels corresponds to the fitting by an Arrhenius law, $1 / \tau_{\mathrm{Ti}-\mathrm{Ti}_{\mathrm{i}}}=v_{\mathrm{O}} \exp \left(-U / \mathrm{k}_{\mathrm{B}} T\right)$.

decreases from $\sim 60 \mathrm{~cm}^{-1}$ to nearly zero when the system is cooled down from $1,000 \mathrm{~K}$ to the lowest investigated temperatures (note that these low frequencies imply that the relaxational $\mathrm{Ti}$ mode does not overlap with the $\mathrm{Zr}$ bare DHO mode, therefore preventing a Fano coupling between these two modes). Figure $4 \mathrm{~b}$ clearly shows that this frequency follows an Arrhenius law, $1 / \tau_{\mathrm{Ti}}$ $\mathrm{Ti}_{\mathrm{i}}=v_{0} \exp \left(-U / k_{\mathrm{B}} T\right)$, with $v_{0} \simeq 2.8 \mathrm{THz}$ and the activation energy, $U \simeq 0.038 \mathrm{eV}$, when the temperature is above $\sim 130 \mathrm{~K}$. Interestingly, a thermally activated process with an activation energy of the same order of magnitude was indeed observed experimentally in the $\mathrm{THz}$ spectra of $\mathrm{Ba}\left(\mathrm{Zr}_{0.4} \mathrm{Ti}_{0.6}\right) \mathrm{O}_{3}$ (ref. 28), which further attests the validity of our simulations (more precisely, the observed $\mathrm{THz}$ relaxation mode has been quantitatively evaluated in an unpublished work by the authors of ref. 28 for temperatures ranging from 300 to $700 \mathrm{~K}$, and can be described by an Arrhenius law with an estimated activation energy of $0.07 \mathrm{eV}$ and a limiting frequency of $1 \mathrm{THz}$ ). It is also worthwhile to note that Fig. 4 seems to indicate that the relaxation time of $R_{\mathrm{Ti}-\mathrm{Ti}}$ deviates from the Arrhenius law below $130 \mathrm{~K}$. This temperature was found in ref. 26 to be the characteristic temperature, $T_{\mathrm{f}}$, that signals the formation of (larger) static PNRs, all having a polarization being aligned along one of the eight possible $\langle 111\rangle$ pseudocubic directions (note that $T_{\mathrm{f}}$ was practically determined to be the temperature at which the static dielectric response peaks, and was termed as the freezing temperature in ref. 26. This denomination of freezing temperature has to be taken with a grain of salt, since the traditional definition of a freezing temperature for relaxors is technically different: it is rather the non-zero temperature appearing in the Vogel-Fulcher law for which the relaxation time tends to be infinite ${ }^{4}$ ). However, one has also to realize that the relaxation frequency of $R_{\mathrm{Ti}-\mathrm{Ti}}$ below $130 \mathrm{~K}$ begins to become close to the limiting minimal values that are technically accessible by our MD simulations (estimated to be $\sim 0.3 \mathrm{~cm}^{-1}$ ). An experimental validation of our prediction of a change in Arrhenius behaviour of $R_{\mathrm{Ti}-\mathrm{Ti}}$ around $T_{\mathrm{f}}$ is therefore needed before asserting that this is not an artefact of the MD simulations. Let us also indicate that, at frequencies below $\mathrm{GHz}$ range (which are technically inaccessible in our simulations), another thermally activated relaxation following the Arrhenius behaviour was experimentally revealed for $T<T_{\mathrm{B}}{ }^{27,28}$. This relaxation was found to be independent of the $\mathrm{Ti}$ concentration in the BZT solid solutions and to possess a somewhat higher activation energy of $U \simeq 0.167 \mathrm{eV}$ (refs 27,28 ). In contrast to $R_{\mathrm{Ti}-\mathrm{Ti}}$ that is associated with reorientations of individual dipoles in Ti cells, we believe that the lowest in-frequency relaxation mode may originate from the collective dynamics inside PNRs or even thermally activated reorientations of the whole PNRs 3,4 .

We now briefly discuss the dielectric response, $\chi^{\mathrm{Ti}, \mathrm{Zr}}$, obtained from the cross-correlation of $\mathrm{Zr}$ and $\mathrm{Ti}$ sites (Fig. 1c). As discussed above, the unusual striking features of $\operatorname{Im}\left(\chi^{\mathrm{Ti}, \mathrm{Zr}}\right)$, including the negative values in some frequency range and its asymmetric lineshape, can be qualitatively well understood by considering a Fano resonance between the bare response of the $\mathrm{Zr}$ sublattice and the bare response inherent to the $\mathrm{Ti}$ sublattice. Practically, the following phenomenological expression (which is adapted from the original equation proposed by Fano himself ${ }^{49}$ ) was found necessary to adequately fit the MD data, in general, and quantitatively reproduce such subtle and fine features, in particular, $Q(v)=Q_{0}(v)\left[\frac{\left(q \gamma_{\text {res }} / 2+v-v_{0}\right)^{2}}{\left(\gamma_{\text {res }} / 2\right)^{2}+\left(v-v_{0}\right)}-1\right]$, where $v_{0}$ is the resonance frequency, $\gamma_{\text {res }}$ describes the linewidth and $q_{\text {res }}$ is the Fano parameter determining the asymmetry of the lineshape of the resonance, which is traditionally understood as a ratio between the rates of two transition pathways: one for the direct transition to the discrete state and the other for the two-step transition to the discrete state via the continuum ${ }^{62}$. Note that the -1 appearing in the bracket is inserted here in order to exclude the background continuum response. Finally, $Q_{0}(v)$ typically represents the unperturbed, but nonuniform, continuum background absorption ${ }^{63} . Q_{0}(v)$ therefore originates from the Ti dipoles and, thus, can be represented by a Debye and a DHO mode, that is,

$$
Q_{0}(v)=\operatorname{Im}\left[\frac{S_{0}}{1-i \tau_{0} v}+\frac{\Omega_{1}^{2}}{v_{1}^{2}-v^{2}-i \gamma_{1} v}\right]
$$

Note that equation (8) represents the absorption spectra of the bare $\mathrm{Ti}$ contribution, that is, the broadband $\mathrm{Ti}$ resonance that is not affected by the interaction with the $\mathrm{Zr}$ sublattice. As a result, the parameters of equation (8) differ from those of equation (7), since the latter describes the perturbed response. The difference between the bare and perturbed responses of the $\mathrm{Ti}$ sublattice is further emphasized when realizing that two DHOs are used in equation (7) versus a single one in equation (8). We numerically found that the fitting of $\chi^{\mathrm{Ti}, \mathrm{Zr}}$ by $Q(v)$ is rather good for all the available temperatures (as shown in Fig. 1c). It is also interesting to point out that modifications of the Fano resonance, which bear 
resemblance to the expressions of $Q(v)$ and $Q_{0}(v)$ here, were also proposed in ref. 52.

In summary, we obtained and analysed the $\mathrm{THz}$ dielectric response of $\mathrm{Ba}\left(\mathrm{Zr}_{0.5} \mathrm{Ti}_{0.5}\right) \mathrm{O}_{3}$ relaxors by decomposing it into its constitutional parts that are defined by equations $(2-4)$. This decomposition and the analysis via the coupled DHO model using Green's function allow us to reveal the existence, at any temperature, of a Fano resonance between the bare discrete, sharp DHO mode associated with the $\mathrm{Zr}$ sublattice and the bare broad DHO mode inherent to the Ti sublattice. Such difference in broadness between the bare DHO modes of the $\mathrm{Zr}$ versus the Ti sublattices originates from the fact that $\mathrm{Zr}$ ions energetically prefer to stay close to their ideal positions while Ti ions want to move off-centre with the amplitude of their motions being influenced by their environment. This difference in off-centering is precisely the reason found in ref. 26 to explain why the disordered BZT possesses PNRs and is a relaxor. It is expected that the presently discovered Fano resonance may also occur in other solid solutions whose relaxor properties arise from such difference in off-centering between their alloyed elements. Note, however, that lead-based relaxors (for example, the $\mathrm{Pb}\left(\mathrm{Mg}_{1 / 3} \mathrm{Nb}_{2 / 3}\right) \mathrm{O}_{3}$-based systems), which are likely more complicated than lead-free relaxors, may need to invoke rather complex phenomena to reproduce their properties ${ }^{64}$. Our simulations also predict a thermally activated $\mathrm{THz}$ relaxation mode, which is in agreement with the previous experiment ${ }^{28}$. Finally, the phonon mode of the $\mathrm{Ti}$ sublattice hardens upon cooling through the $T^{*}$ temperature that is known to exist in relaxors. We therefore hope that the present work is beneficial to better understand relaxors.

\section{Methods}

Effective Hamiltonian. The effective Hamiltonian for BZT used here has been developed in ref. 26 and has been shown to reproduce many finite-temperature relaxor properties of this material ${ }^{26,30,31}$. Its internal energy is:

$$
E=E_{\text {int }}\left(\left\{\mathbf{u}_{i}\right\},\left\{\sigma_{j}\right\},\left\{\mathbf{v}_{i}\right\}, \eta_{\mathrm{H}}\right),
$$

where $\mathbf{u}_{i}$ is the local soft-mode in unit cell $i$, which is directly proportional to the local electric dipole moment in that cell. $\mathbf{u}_{i}$ is centred on the B-sites $(\mathrm{Zr}$ or $\mathrm{Ti}$ ) and represents the collective motion of $\mathrm{Ba}, \mathrm{Zr} / \mathrm{Ti}$ and oxygen atoms inside this cell. $\left\{\sigma_{j}\right\}$ characterizes the atomic arrangement, that is $\sigma_{j}=+1$ or -1 corresponds to the presence of a $\mathrm{Zr}$ or $\mathrm{Ti}$ atom located at the lattice site $j$, respectively ${ }^{65}$. The v's are Ba-centred dimensionless local displacements that are related to the inhomogeneous strain inside each cell ${ }^{66} . \eta_{\mathrm{H}}$ is the homogeneous strain tensor ${ }^{66}$. The internal energy of equation (9) has two parts. The first part mimics the energetics of an hypothetical, simple $\mathrm{Ba}\langle B\rangle \mathrm{O}_{3}$ crystal in which $\langle B\rangle$ is a virtual atom that can be thought as a chemical average of $\mathrm{Zr}$ and $\mathrm{Ti}$ atoms ${ }^{65-67}$. This first term collects energies related to local mode self-energy, short-range and long-range interactions between local modes, as well as elastic energy and the couplings between local modes and strains ${ }^{66}$. The second part is explicitly related to alloy effects on physical properties. It includes the on-site effects resulting from the difference in harmonicity between the $\mathrm{Zr}$ and $\mathrm{Ti}$ ions (this difference was found to be crucial to reproduce and understand relaxor behaviour in $\mathrm{BZT}^{26,30}$ ), as well as intersite effects such as random fields and strains arising from the distribution of the $\mathrm{Zr}$ and $\mathrm{Ti}$ ions inside the supercell. All the parameters entering the analytical expression of $E_{\text {int }}$ are determined by performing first-principle calculations ${ }^{67-70}$ on relatively small supercells (typically, $<20$ atoms). Practically, the PNRs are obtained by using a computer programme that examines the dipole at each Ti site to determine whether it belongs to a $\mathrm{PNR}^{30,31}$.

Molecular dynamics. In order to obtain the dynamical properties of BZT, the $E_{\text {int }}$ internal energy of equation (9) is used in MD simulations (rather than in Monte-Carlo simulations as in refs 26,30,31), with its degrees of freedom obeying Newton's equation ${ }^{56}$ :

$$
M_{A_{\gamma}} \frac{\mathrm{d}^{2}}{\mathrm{~d} t^{2}} A_{\gamma}=-\frac{\partial}{\partial A_{\gamma}} E_{\text {int }}
$$

where $A_{\gamma}$ is one (Cartesian or Voigt) component of the aforementioned local modes or strains and $M_{A \gamma}$ has a dimension of a mass. During the MD simulations, the temperatures of all these structural degrees of freedom are controlled by EvansHoover thermostats ${ }^{56,59}$. For each MD simulation, we first run $10^{5} \mathrm{MD}$ steps of NPT (isothermal-isobaric ensemble) simulations to equilibrate the system at a chosen temperature and pressure. Then, the equilibration of the system within an NVE (microcanonical) ensemble is performed through $10^{5} \mathrm{MD}$ steps. Subsequent $4.3 \times 10^{6} \mathrm{NVE}$ steps are performed to obtain the time-resolved physical properties of the system.

\section{References}

1. Smolensky, G. A. Ferroelectrics and Related Materials (Gordon and Breach, 1981).

2. Burns, G. \& Dacol, F. H. Crystalline ferroelectrics with glassy polarization behavior. Phys. Rev. B 28, 2527-2530 (1983).

3. Cross, L. E. Relaxor ferroelectrics. Ferroelectrics 76, 241-267 (1987).

4. Viehland, D., Jang, S. J., Cross, L. E. \& Wuttig, M. Freezing of the polarization fluctuations in lead magnesium niobate relaxors. J. Appl. Phys. 68, 2916 (1990)

5. Westphal, V., Kleemann, W. \& Glinchuk, M. Diffuse phase transitions and random-field-induced domain states of the 'relaxor' ferroelectric $\mathrm{PbMg}_{1 / 3} \mathrm{Nb}_{2 / 3} \mathrm{O}_{3}$. Phys. Rev. Lett. 68, 847-850 (1992).

6. Colla, E. V., Koroleva, E. Y., Okuneva, N. M. \& Vakhrushev, S. B. Long-time relaxation of the dielectric response in lead magnoniobate. Phys. Rev. Lett. $\mathbf{7 4}$ 1681-1684 (1995).

7. Tagantsev, A. K. \& Glazounov, A. E. Mechanism of polarization response in the ergodic phase of a relaxor ferroelectric. Phys. Rev. B 57, 18-21 (1998).

8. Pirc, R. \& Blinc, R. Spherical random-bond-random-field model of relaxor ferroelectrics. Phys. Rev. B 60, 13470-13478 (1999).

9. Vugmeister, B. \& Rabitz, H. Dynamics of interacting clusters and dielectric response in relaxor ferroelectrics. Phys. Rev. B 57, 7581-7585 (1998).

10. Levstik, A., Kutnjak, Z., Filipič, C. \& Pirc, R. Glassy freezing in relaxor ferroelectric lead magnesium niobate. Phys. Rev. B 57, 11204-11211 (1998).

11. Takesue, N., Fujii, Y., Ichihara, M. \& Chen, H. Self-accommodation of ionic size-effect atomic displacements in antiferroelectric order in relaxor lead scandium niobate. Phys. Rev. Lett. 82, 3709 (1999).

12. Kutnjak, Z., Filipič, C., Pirc, R. \& Levstik, A. Slow dynamics and ergodicity breaking in a lanthanum-modified lead zirconate titanate relaxor system. Phys. Rev. B 59, 294-301 (1999).

13. Xu, G., Shirane, G., Copley, J. \& Gehring, P. Neutron elastic diffuse scattering study of $\mathrm{Pb}\left(\mathrm{Mg}_{1 / 3} \mathrm{Nb}_{2 / 3}\right) \mathrm{O}_{3}$. Phys. Rev. B 69, 064112 (2004).

14. Jeong, I.-K. et al. Direct observation of the formation of polar nanoregions in $\mathrm{Pb}\left(\mathrm{Mg}_{1 / 3} \mathrm{Nb}_{2 / 3}\right) \mathrm{O}_{3}$ using neutron pair distribution function analysis. Phys. Rev. Lett. 94, 147602 (2005).

15. Svitelskiy, O., La-Orauttapong, D. \& Toulouse, J. $\mathrm{PbTiO}_{3}$ addition and interna dynamics in $\mathrm{Pb}\left(\mathrm{Zn}_{1 / 3} \mathrm{Nb}_{2 / 3}\right) \mathrm{O}_{3}$ crystal studied by Raman spectroscopy. Phys. Rev. B 72, 172106 (2005)

16. Ishchuk, V., Baumer, V. N. \& Sobolev, V. L. The influence of the coexistence of ferroelectric and antiferroelectric states on the lead lanthanum zirconate titanate crystal structure. J. Phys.: Condens. Matter 17, L177-L182 (2005)

17. Bokov, A. A. \& Ye, Z.-G. Recent progress in relaxor ferroelectrics with perovskite structure. J. Mater. Sci. 41, 31-52 (2006).

18. Tinte, S., Burton, B. P., Cockayne, E. \& Waghmare, U. V. Origin of the relaxor state in $\mathrm{Pb}\left(\mathrm{B}_{x}, \mathrm{~B}_{1-x}\right) \mathrm{O}_{3}$ perovskites. Phys. Rev. Lett. 97, 137601 (2006).

19. Bokov, A. A., Maglione, M. \& Ye, Z.-G. Quasi-ferroelectric state in Ba $\left(\mathrm{Ti}_{1-x} \mathrm{Zr}_{x}\right) \mathrm{O}_{3}$ relaxor: dielectric spectroscopy evidence. J. Phys.: Condens. Matter 19, 092001 (2007).

20. Grinberg, I., Juhas, P., Davies, P. K. \& Rappe, A. M. Relationship between local structure and relaxor behavior in perovskite oxides. Phys. Rev. Lett. 99, 267603 (2007).

21. Grinberg, I., Shin, Y.-H. \& Rappe, A. M. Molecular dynamics study of dielectric response in a relaxor ferroelectric. Phys. Rev. Lett. 103, 197601 (2009).

22. Bai, Y. \& Jin, L. Characterization of frequency-dependent glass transition temperature by Vogel-Fulcher relationship. J. Phys. D Appl. Phys. 41, 152008 (2008).

23. Toulouse, J. \& Pattnaik, R. K. Pretransitional condensation in mixed ferrolectrics. J. Phys. Chem. Solids 57, 1473 (1996).

24. Dkhil, B. et al. Intermediate temperature scale $T^{\star}$ in lead-based relaxor systems. Phys. Rev. B 80, 064103 (2009).

25. Al-Zein, A., Hlinka, J., Rouquette, J. \& Hehlen, B. Soft mode doublet in $\mathrm{PbMg}_{1 / 3} \mathrm{Nb}_{2 / 3} \mathrm{O}_{3}$ relaxor investigated with hyper-raman scattering. Phys. Rev. Lett. 105, 017601 (2010).

26. Akbarzadeh, A., Prosandeev, S., Walter, E., Al-Barakaty, A. \& Bellaiche, L. Finite-temperature properties of $\mathrm{Ba}(\mathrm{Zr}, \mathrm{Ti}) \mathrm{O}_{3}$ relaxors from first principles. Phys. Rev. Lett. 108, 257601 (2012).

27. Nuzhnyy, D. et al. Broadband dielectric response of $\mathrm{Ba}(\mathrm{Zr}, \mathrm{Ti}) \mathrm{O}_{3}$ ceramics: from incipient via relaxor and diffuse up to classical ferroelectric behavior. Phys. Rev. B 86, 014106 (2012).

28. Petzelt, J. et al. Broadband dielectric spectroscopy of $\mathrm{Ba}(\mathrm{Zr}, \mathrm{Ti}) \mathrm{O}_{3}$ : dynamics of relaxors and diffuse ferroelectrics. Ferroelectrics 469, 14-25 (2014).

29. Kleemann, W. Random fields in relaxor ferroelectrics-a jubilee review. J. $A d v$. Dielect. 2, 12410001 (2012) 
30. Prosandeev, S., Wang, D., Akbarzadeh, A., Dkhil, B. \& Bellaiche, L. Fieldinduced percolation of polar nanoregions in relaxor ferroelectrics. Phys. Rev. Lett. 110, 207601 (2013).

31. Prosandeev, S., Wang, D. \& Bellaiche, L. Properties of epitaxial films made of relaxor ferroelectrics. Phys. Rev. Lett. 111, 247602 (2013).

32. Lebedev, A. Properties of $\mathrm{BaTiO}_{3} / \mathrm{BaZrO}_{3}$ ferroelectric superlattices with competing instabilities. Phys. Solid State 55, 1198-1206 (2013).

33. Gehring, P. M. Neutron diffuse scattering in lead-based relaxor ferroelectrics and its relationship to the ultra-high piezoelectricity. J. Adv. Dielect. 2, 1241005 (2012).

34. Cowley, R. A., Gvasaliya, S. N., Lushnikov, S. G., Roessli, B. \& Rotaru, G. M. Relaxing with relaxors: a review of relaxor ferroelectrics. Adv. Phys. 60, 229-327 (2011).

35 . Toulouse, J. The three characteristic temperatures of relaxor dynamics and their meaning. Ferroelectrics 369, 203-213 (2008).

36. Laulhé, C. et al. EXAFS study of lead-free relaxor ferroelectric $\mathrm{Ba}\left(\mathrm{Ti}_{1-x} \mathrm{Zr}_{x}\right) \mathrm{O}_{3}$ at the Zr K edge. Phys. Rev. B 74, 014106 (2006).

37. Ke, S. \& Fan, H. Dielectric dispersion behavior of $\mathrm{Ba}(\mathrm{Zr}, \mathrm{Ti}) \mathrm{O}_{3}$ solid solutions with a quasiferroelectric state. J. Appl. Phys. 104, 034108 (2008).

38. Laulhé, C., Pasturel, A., Hippert, F. \& Kreisel, J. Random local strain effects in homovalent-substituted relaxor ferroelectrics: a first-principles study of $\mathrm{BaTi}_{0.74} \mathrm{Zr}_{0.26} \mathrm{O}_{3}$. Phys. Rev. B 82, 132102 (2010).

39. Kholkin, A. \& Morozovska, A. Surface domain structures and mesoscopic phase transition in relaxor ferroelectrics. Adv. Funct. Mater. 21, 1977-1987 (2011).

40. Xie, L. et al. Static and dynamic polar nanoregions in relaxor ferroelectric $\mathrm{Ba}\left(\mathrm{Ti}_{1-x} \mathrm{Sn}_{x}\right) \mathrm{O}_{3}$ system at high temperature. Phys. Rev. B 85, 014118 (2012)

41. Sherrington, D. BZT: a soft pseudo-spin glass. Phys. Rev. Lett. 111, 227601 (2013).

42. Dmowski, W. et al. Local lattice dynamics and the origin of the relaxor ferroelectric behavior. Phys. Rev. Lett. 100, 137602 (2008).

43. Takenaka, H., Grinberg, I. \& Rappe, A. M. Anisotropic local correlations and dynamics in a relaxor ferroelectric. Phys. Rev. Lett. 110, 147602 (2013).

44. Maiti, T., Guo, R. \& Bhalla, A. S. Structure-property phase diagram of $\mathrm{Ba}\left(\mathrm{Zr}_{x} \mathrm{Ti}_{1-x}\right) \mathrm{O}_{3}$ system. J. Amer. Ceram. Soc. 91, 1769-1780 (2008).

45. Simon, A., Ravez, J. \& Maglione, M. The crossover from a ferroelectric to a relaxor state in lead-free solid solutions. J. Phys. Condens. Matter 16, 963-970 (2004).

46. Dixit, A., Majumder, S. B., Katiyar, R. S. \& Bhalla, A. S. Studies on the relaxor behavior of sol-gel derived $\mathrm{Ba}\left(\mathrm{Zr}_{x} \mathrm{Ti}_{1-x}\right) \mathrm{O}_{3}(0.30 \leq x \leq 0.70)$ thin films. J. Mater. Sci. 41, 87-96 (2006).

47. Liu, Y., Withers, R., Nguyen, B. \& Elliott, K. Structurally frustrated polar nanoregions in $\mathrm{BaTiO}_{3}$-based relaxor ferroelectric systems. Appl. Phys. Lett. 91, 152907 (2007).

48. Shvartsman, V. V., Zhai, J. \& Kleemann, W. The dielectric relaxation in solid solutions $\mathrm{BaTi}_{1-x} \mathrm{Zr}_{x} \mathrm{O}_{3}$. Ferroelectrics 379, 77-85 (2009).

49. Fano, U. Effects of configuration interaction on intensities and phase shifts. Phys. Rev. 124, 1866 (1961).

50. Miroshnichenko, A. E., Flach, S. \& Kivshar, Y. S. Fano resonances in nanoscale structures. Rev. Mod. Phys. 82, 2257-2298 (2010).

51. Zhang, Y., Hu, X., Fu, Y., Yang, H. \& Gong, Q. Ultrafast all-optical tunable Fano resonance in nonlinear ferroelectric photonic crystals. Appl. Phys. Lett. 100, 031106 (2012).

52. Gallinet, B. \& Martin, O. J. F. Ab initio of Fano resonances in plasmonic nanostructures and metametrials. Phys. Rev. B 83, 235427 (2011).

53. Toulouse, J., Cai, L., Pattnaik, R. K. \& Boatner, L. A. Multiscale dynamics in relaxor ferroelectrics. Europhys. Lett. 105, 17001 (2014).

54. Wang, D. et al. Fermi resonance involving nonlinear dynamical couplings in $\mathrm{Pb}(\mathrm{Zr}, \mathrm{Ti}) \mathrm{O}_{3}$ solid solutions. Phys. Rev. Lett. 107, 175502 (2011).

55. Weerasinghe, J., Wang, D. \& Bellaiche, L. Low-frequency coupled modes in disordered $\mathrm{Pb}(\mathrm{Zr}, \mathrm{Ti}) \mathrm{O}_{3}$ solid solutions from first principles. Phys. Rev. B 85, 014301 (2012)

56. Ponomareva, I., Bellaiche, L., Ostapchuk, T., Hlinka, J. \& Petzelt, J. Terahertz dielectric response of cubic $\mathrm{BaTiO}_{3}$. Phys. Rev. B 77, 012102 (2008).

57. Hlinka, J., Ostapchuk, T., Nuzhnyy, D. \& Petzelt, J. Coexistence of the phonon and relaxation soft modes in the terahertz dielectric response of tetragonal $\mathrm{BaTiO}_{3}$. Phys. Rev. Lett. 101, 167402 (2008).
58. Caillol, J. M., Levesque, D. \& Weis, J. J. Theoretical calculation of ionic solution properties. J. Chem. Phys. 85, 6645-6657 (1986).

59. Wang, D., Weerasinghe, J., Albarkati, A. \& Bellaiche, L. Terahertz dielectric response and coupled dynamics of ferroelectrics and multiferroics from effective Hamiltonian simulations. Inter. J. Mod. Phys. B 27, 1330016 (2013).

60. Barker, A. S. \& Hopfield, J. J. Coupled-Optical-Phonon-Mode Theory of the Infrared Dispersion in $\mathrm{BaTiO}_{3}, \mathrm{SrTiO}_{3}$, and $\mathrm{KTaO}_{3}$. Phys. Rev 135, A1732 (1964).

61. Hlinka, J., Petzelt, J., Kamba, S., Noujni, D. \& Ostapchuk, P. Infrared dielectric response of relaxor ferroelectrics. Phase Trans. 79, 41-78 (2006).

62. Rau, A. R. P. Perspectives on the Fano Resonance Formula. Phys. Scr. 69, C10 (2004).

63. Lim, J. et al. Effect of nonuniform continuum density of states on a Fano resonance in semiconductor quantum wells. Phys. Rev. B 80, 035322 (2009).

64. Manley, M. E. et al. Phonon localization drives polar nanoregions in a relaxor ferroelectric. Nat. Commun. 5, 3683 (2014).

65. Bellaiche, L., García, A. \& Vanderbilt, D. Finite-temperature properties of $\mathrm{Pb}\left(\mathrm{Zr}_{1-x} \mathrm{Ti}_{x}\right) \mathrm{O}_{3}$ alloys from first principles. Phys. Rev. Lett. 5427-5430 (2000).

66. Zhong, W., Vanderbilt, D. \& Rabe, K. M. First-principles theory of ferroelectric phase transitions for perovskites: The case of $\mathrm{BaTiO}_{3}$. Phys. Rev. B 52, 6301-6312 (1995).

67. Bellaiche, L. \& Vanderbilt, D. Virtual crystal approximation revisited: application to dielectric and piezoelectric properties of perovskites. Phys. Rev. B 61, 7877-7882 (2000).

68. Hohenberg, P. \& Kohn, W. Inhomogeneous electron gas. Phys. Rev. 136, B864 (1964).

69. Kohn, W. \& Sham, L. Self-consistent equations including exchange and correlation effects. Phys. Rev. 140, A1133 (1965).

70. Vanderbilt, D. Soft self-consistent pseudopotentials in a generalized eigenvalue formalism. Phys. Rev. B 41, 7892 (1990).

\section{Acknowledgements}

Discussions with Profs. C.-L. Wang, X.-Y. Wei, and C.-L. Jia are greatly acknowledged. This work is financially supported by the National Science Foundation (NSF) grant DMR-1066158 (D.W. and L.B.). P.O., J.P. and J.H. acknowledge the support from the Czech Science foundation (Project 13-15110S). Z.G.Y. and A.A.B. thank the support from the Natural Science and Engineering Research Council of Canada (NSERC). Z.G.Y also acknowledges the support from Office of Naval Research grant N00014-12-1-1045, and the International Science \& Technology Cooperation Program of China (Grant No. 2011DFA51880), the National Natural Science Foundation of China (NSFC) grants 50728201 and 90923001, and the 'Qianren Program' of the Chinese Government. D.W also acknowledges the support from the NSFC grant 51390472 and the 111 Project (B14040). Some computations were made possible thanks to the grants 0722625 , 0959124, and 0918970 from NSF.

\section{Author contributions}

D.W. and L.B. conceived and designed this study. D.W. performed molecular dynamics simulations. D.W., P.O. and J.H. analysed the dielectric responses, and contributed to the design of the fitting scheme of the data. D.W., J.H., A.A.B. and L.B. wrote the first draft of the manuscript. All authors contributed extensively to the interpretation of the results and to the final version of the manuscript.

\section{Additional information}

Supplementary Information accompanies this paper at http://www.nature.com/ naturecommunications

\section{Competing financial interests: The authors declare no competing financial interests}

Reprints and permission information is available online at http://npg.nature.com/ reprintsandpermissions/

How to cite this article: Wang, D. et al. Fano resonance and dipolar relaxation in lead-free relaxors. Nat. Commun. 5:5100 doi: 10.1038/ncomms6100 (2014). 